\title{
AN EFFICIENCY METER FOR ELECTRIC INCANDESCENT LAMPS.
}

\author{
By Edward P. Hyde and H. B. Brooks.
}

\section{INTRODUCTION.}

The life of a normal incandescent lamp depends primarily on the temperature at which the lamp is operated, and the temperature of the filament is a function, not of the watts or of the candlepower simply, but of the ratio, watts per candle; that is, of the so-called efficiency ${ }^{1}$ at which the lamp is operated. For lamps of the same type, the higher the efficiency the shorter the life, and vice versa; and since uniformity in life is one of the first qualifications of a good lot of lamps, uniformity in efficiency is of great importance.

There is at present no direct reading "efficiency meter" on the market, so far as the writers know, and it may be that this is partly the reason for the present custom of specifying limits of watts and candlepower, and not limits of efficiency. In order to meet the need for an efficiency meter in the photometric work of the Bureau of Standards, the writers have recently designed and constructed such an instrument, the theory and operation of which will be described in this paper.

\footnotetext{
"In the following paper the term "efficiency" will be used to designate the watts per mean horizontal candle, in accordance with the more common use of the term in industrial practice. The proper definition of the word "efficiency" would be lumens per watt, which gives a much more significant constant for a radiating source than watts per mean horizontal candle. For incandescent lamps of the same type, however, in which the ratio of mean spherical to mean horizontal intensity is approximately constant for different lamps, the watts per mean horizontal candle gives an approximate idea of the relative temperatures of the filaments. Moreover, all that is said here in regard to watts per mean horizontal candle would apply equally well to watts per mean spherical candle, if the photometer gave readings of mean spherical instead of mean horizontal intensity, and by adding a new scale to the wattmeter direct readings in lumens per watt could be made.
} 
The problem to be solved consisted in devising some method by which the readings of a wattmeter would be made to vary inversely as the candlepower. Two general methods suggested themselves, a mechanical method and an electrical one. According to the former either the scale of the wattmeter would be moved by the motion of the photometer carriage, or the motion of the latter would vary the counterforce opposed to the moving coil, as, for example, by increasing or decreasing the effective length of the spring. Either of these methods would require the wattmeter to be specially constructed, and to be fixed in position relative to the photometer, and would present other serious practical difficulties, so that the mechanical method was abandoned for the more promising electrical method.

According to the latter there are three possible ways of decreasing the wattmeter reading in the ratio of $\frac{\text { Constant }}{\text { Candlepower }}:(a)$ by placing a variable resistance in parallel with the current coil of the wattmeter; $(b)$ by placing a variable resistance in parallel with the moving coil; and $(c)$ by placing a variable resistance in series with the pressure circuit in the wattmeter. Before discussing these methods it should be pointed out that in every case this variable resistance must be controlled by the motion of the photometer, as by the use of a sliding contact moving over a coil, so that for all positions of the photometer the reading of the instrument shall be

$$
\text { Constant } \times \frac{\text { Watts }}{\text { Candlepower }}
$$

where the candlepower is given by the index on the photometer carriage. If we should make the constant equal to unity the reading on the instrument would be so small compared with the full scale deflection that only a relatively low accuracy could be obtained, so that it seemed desirable to make the constant Io, since then the reading would come at a good point on the scale and at the same time the scale reading would give the true watts per candle by moving the decimal point. To take an example, suppose that a I6-cp lamp requires 64 watts at normal voltage. In order to make the instrument read watts per candle directly we must so alter the resistance of the instrument by one of the above methods, or by 
some combination of them, that the instrument shall indicate I I6 of its original reading; that is, $64 \times 6=4$. If, however, instead of reducing the reading to I I 6 we reduce it to Io 16 of its original value, it will now give a reading of $10 \mathrm{I} 6 \times 64=40$, which will be ten times the watts per candle, but which will come at a much more desirable part of the scale of the instrument. At Io $\mathrm{cp}$ the instrument will read Io Io $\times$ watts, or watts directly, at $20 \mathrm{cp}$ Io $20 \times$ watts or one-half the watts, and so on for other candlepower values.

To return to the three electrical methods suggested for the reduction of the wattmeter reading, case $(a)$ is objectionable because of the low resistance of the series coil in a wattmeter of the required range. It would necessitate a low resistance in the shunt circuit controlled by the moving screen, and hence would be liable to error due to variations in the contact resistance. In case $(b)$ this objection will not arise, since the resistance of the moving coil will be about 50 ohms, and hence the shunt controlled by the movement of the screen will not, in general, be less than 50 ohms, in comparison with which variations in the resistance of well made sliding contacts will be negligible. This method requires that an extra lead be brought out to an extra binding post on the wattmeter, as both terminals of the moving coil are not directly accessible.

In both the shunt methods the range of the efficiency meter, in case the instrument is to read ro times the efficiency, will have as its lower limit Io $\mathrm{cp}$, for at Io $\mathrm{cp}$ the resistance of the external shunt must be infinite, or, in other words, the shunt circuit must be open. Moreover, in case $(b)$ if the instrument is to be used for candle-power values above $20 \mathrm{cp}$ the shunt resistance would become smaller than 50 ohms, and hence care must be exercised to have the contact resistance sufficiently constant to produce no appreciable error. In general practice, however, in reading I6-cp lamps the range is seldom greater than from Io to $20 \mathrm{cp}$, so that these difficulties are not serious.

Method $(c)$ is more nearly free from errors and limitations than either of the other two methods. According to this method a variable resistance is placed in series with the pressure circuit in the wattmeter, and since the latter will have a resistance of several thousand ohms, the contact resistance is of much less importance than in the other cases. Moreover there are practically no limits to the range of the instrument, if we take out of the wattmeter part of the resist- 
ance in series with the moving coil. This becomes necessary only in case we desire to use the efficiency meter below Io cp; otherwise the wattmeter can be used without any modification. Method $(c)$ therefore seemed more promising than either of the other two methods, and was hence adopted.

\section{THEORY OF METHOD.}

Assuming that the wattmeter to be used reads correctly as a wattmeter, the resistance in series with it should be zero at ro cp, since the reading at Io $\mathrm{cp}$ should be total watts. At $20 \mathrm{cp}$ the reading should be one-half the total watts; that is, the added resistance for the position of the photometer corresponding to $20 \mathrm{cp}$ should equal the resistance of the wattmeter. It only remains, therefore, to determine the relation between the candle-power reading and the resistance for other points on the bar, and to find some convenient practical way of introducing the additional resistance.

Let $2 a$ be the distance (supposed to be constant) between the two lamps, $I_{1}$ the intensity of the comparison lamp, $I$ the intensity of the test lamp, and $x$ the distance from the test lamp to the photometer screen when in a position of balance. Then by the inverse square law,

$$
I=I_{1}\left(\frac{x}{2 a-x}\right)^{2}
$$

Moreover, if $W$ is the total watts supplied to the lamp, and $E$ the efficiency or watts per candle,

$$
E=\frac{W}{I}
$$

and if $S$ denote the scale reading of the instrument when the external resistance $r$ is in series with the wattmeter pressure winding of resistance $r_{1}$, and $K$ the factor of proportionality between the scale reading and the efficiency, we get

$$
S=\frac{r_{1}}{r_{1}+r} W=K E=K \frac{W}{I}
$$

or

$$
\frac{r_{1}}{r_{1}+r}=\frac{K}{I}
$$


from which

$$
r=r_{1}\left(\frac{I}{K}-\mathrm{I}\right)
$$

Substituting for $I$ its value as given in equation (I),

$$
r=r_{1}\left[\frac{I_{1}}{K}\left(\frac{x}{2 a-x}\right)^{2}-\mathrm{I}\right]
$$

which gives the resistance $r$, which must be added to the resistance $r_{1}$ for any position $x$ on the bar.

The method which seemed most desirable for the construction of a rheostat to follow this law consisted in winding closely a fine silkcovered resistance wire on a block of insulating material. After coating with shellac varnish and drying, the insulation was removed along a strip over which travels a contact brush attached to the photometer carriage. The variable rheostat thus formed is connected in the pressure circuit of the wattmeter, so that the resistance of this circuit varies with the position of the photometer. The electrical connections are shown diagram-

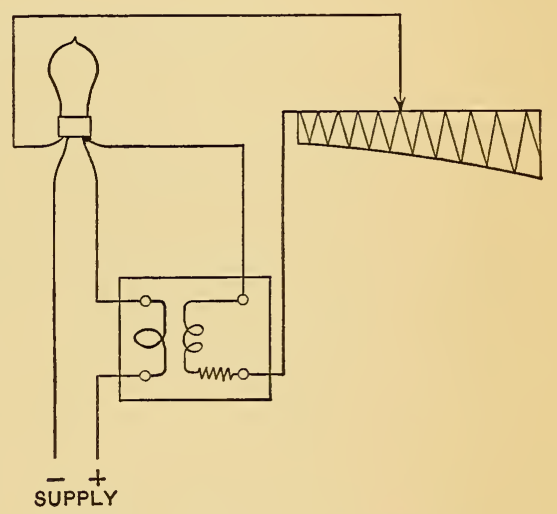

Fig. 1.-Diagram of Connections. matically in Fig. I. The top of the block, along which the contact is made, is straight, and the bottom of the block must have whatever form is necessary to give the proper increments $d r$ to the resistance as the contact moves over successive small distances $d x$. By differentiating equation (6) with respect to $x$ we get the equation,

$$
\frac{d r}{d x}=\frac{4 a r_{1} I_{1}}{K} \frac{x}{(2 a-x)^{3}}
$$

If we let $h$ be the thickness of the block, $y$ the height of the block at any point-that is, the ordinate of the curve measured downward$\rho$ the specific resistance of the wire, $2 b$ the diameter of the bare wire, 
and $n$ the number of turns of the covered wire per unit distance on the block, then the resistance $d r$ contained in a small distance $d x$ is

$$
d r=\frac{2(y+h) \rho}{\pi b^{2}} \times n d x
$$

or

$$
\frac{d r}{d x}=\frac{2 n \rho}{\pi b^{2}}(y+h)
$$

Equating this value of $\frac{d r}{d x}$ to that obtained in equation (7)

from which

$$
\frac{2 n \rho}{\pi b^{2}}(y+h)=\frac{4 a r_{1} I_{1}}{K} \frac{x}{(2 a-x)^{3}}
$$

$$
y=\frac{2 \pi a b^{2} r_{1} I_{1}}{K n \rho} \frac{x}{(2 a-x)^{3}}-h,
$$

which relation determines the form of the bottom of the block. In this equation $a, r_{1}, I_{1}, K$, and $h$ may be taken as constants, while $b$, $n$, and $\rho$ depend on the wire that is used. We may introduce the resistance per unit length, $\rho_{1}$, instead of the specific resistance $\rho$, using the relation

$$
\rho_{1}=\frac{\rho}{\pi b^{2}}
$$

Combining this with equation (II) we get the more convenient form

$$
y=\frac{2 a r_{1} I_{1}}{K n \rho_{1}} \frac{x}{(2 a-x)^{3}}-h,
$$

involving the two wire constants $n$ and $\rho_{1}$. If then a block of insulating material is constructed, having the general form indicated in Fig. 2, the curvature of the bottom conforming to equation (I2), and if the wire whose constants are used in equation (I2) is wound closely on this block, the variable rheostat thus formed, if put in the pressure circuit of the wattmeter, as indicated in Fig. I, will cut down the reading of the wattmeter for all positions of the photometer within the limits of the coil, so that the instrument will indicate, not watts, but a constant $K$ times the efficiency of the lamp. 


\section{CONSTRUCTION OF INSTRUMENT.}

It has been shown that it is desirable to give the constant $K$ the value Io, since then the wattmeter reads watts per candle directly, by moving the decimal point. We will assume that the apparatus is to be designed for r6-cp lamps. In adjusting a photometer for the measurement of I6-cp lamps the voltage on the comparison lamp is adjusted until the reading on a r6-cp standard lamp placed in the test-lamp socket comes at $16 \mathrm{cp}$, the middle point on the bar. Then the intensity of the comparison lamp, $I_{1}$, is $16 \mathrm{cp}$. The distance between the two lamps in commercial photometry is always constant and equal to some standard length, such as IOO or 200 inches, or $250 \mathrm{~cm}$. Hence $a$, which is one-half this distance, is the constant distance from either lamp to the center of the bar. In the commercial photometer at the Bureau of Standards $\alpha=125 \mathrm{~cm}$. A Weston wattmeter was used having a resistance of $2,630 \mathrm{ohms}$. The other constants entering into equation (I2) are $h$, the thickness of the block, which was made I cm, and the two wire constants $n$ and $\rho_{1}$. In designing the resistance block it is necessary to work both ways from equation (I2). First having decided what the approximate dimensions of the block shall be, put in equation (I2) the value of $y$ for some value of $x$, preferably that corresponding to $20 \mathrm{cp}$, and from this equation determine the product $n \rho_{1}$. Then select some wire having approximately this value of $n \rho_{1}$, put the exact values, as obtained from measurements of a sample of the wire, back into equation (I 2), and plot the desired curve.

It may be well to say a word at this point in regard to the limits of the efficiency meter in practice. As stated, if we make $K=$ Io there will be no resistance in series with the wattmeter when the photometer is at ro cp. Hence the coil begins at this point unless it is desired to read efficiencies for points below ro $\mathrm{cp}$, in which case some of the resistance in series with the moving coil can be taken out of the wattmeter and placed in the external coil. This can be accomplished without unfitting the wattmeter for other 11ses, by bringing to an extra binding post on the instrument a tap from some point in the resistance depending on how far below Io $\mathrm{cp}$ the meter is to be used. While there is no difficulty in doing this, there is also no need generally of having the meter read below Io $\mathrm{cp}$ on a I 6 
cp bench. The upper limit can be as high as desired, although the ordinates of the block increase very rapidly as we reach higher candle-power positions, so that unless there is some particular reason to the contrary it will be found desirable to take as the upper limit 20 or $25 \mathrm{cp}$. The meter at the Bureau reads watts per candle for a range of from 10 to $20 \mathrm{cp}$.

We have spoken thus far of a single block wound with wire; we have found it very desirable, however, to have two blocks instead of one. First, for any wire large enough for mechanical strength the slope of the curve at $20 \mathrm{cp}$ is so great that it not only makes an awkward-looking device, but also introduces difficulties in the winding. By using two blocks side by side, equal in every way, the curvature of each is much less than that of the single block. Secondly, with the single block the sliding contact that moves with the photometer must carry with it a flexible wire, which is objectionable. If two blocks are used, however, the photometer merely carries a brush which spans the gap between the two coils. In this arrangement the ends of the wire at the 20-cp point are joined together, keeping the wattmeter circuit closed in case the contact between the brush and the coil should be momentarily broken.

For two blocks of the same thickness the values of $y+h$ deduced from equation (I2) must be divided by 2 , so that

or

$$
y+h=\frac{1}{2} \times \frac{2 a r_{1} I_{1}}{K n \rho_{1}} \frac{x}{(2 a-x)^{3}}
$$

If we put

$$
y=\frac{a r_{1} I_{1}}{\operatorname{Kn} \rho_{1}} \frac{x}{(2 a-x)^{3}}-h
$$

we get

$$
C=\frac{a r_{1} I_{1}}{K n \rho_{1}}
$$

$$
y=C \frac{x}{(2 a-x)^{3}}-h
$$

where $C$ is a constant depending on the values chosen for $n$ and $\rho_{1}$. "Climax" wire was used on account of its high specific resistance, which allows the use of the largest possible wire for a given size of block. The size of the wire was No. 30 B. \& S., and $n$ and $\rho_{1}$ were 
33 turns per $\mathrm{cm}$, and $0.1623 \mathrm{ohm}$ per $\mathrm{cm}$, respectively. Substituting the values in equation (14), and putting for the other constants the values given above,

$$
\begin{aligned}
K & =\mathrm{I0} \\
a & =\mathrm{I} 25 \\
r_{1} & =2630 \\
I_{1} & =\mathrm{I} 6
\end{aligned}
$$

we get

$$
\begin{gathered}
C=\frac{\mathrm{I} 25 \times 2630 \times 16}{\mathrm{IO} \times 33 \times 0.1623} \\
=9.82 \times 10^{4}
\end{gathered}
$$

Putting this value for $C$ in equation (I4),

$$
y=9.82 \times \operatorname{IO}^{4} \times \frac{x}{(250-x)^{3}}-h .
$$

As the edges of the block must be rounded for better winding, the effective value of $h$ is slightly reduced, and a suitable allowance must be made. In equation (I6) $x$ is the distance along the bar measured from the test lamp, and is determined for any candlepower, $I$, by the equation

from which

$$
\frac{I}{I_{1}}=\frac{I}{16}=\frac{x^{2}}{(250-x)^{2}}
$$

$$
x=\frac{250 \sqrt{I}}{\sqrt{I}+4}
$$

From equations (I8) and (I6) we get the numerical values:

$$
\begin{array}{lll}
I=\mathrm{IO} \mathrm{cp} & x=\mathrm{I} I 0.38 \mathrm{~cm} & y=3.08 \mathrm{~cm} \\
I=\mathrm{I} 6 \mathrm{cp} & x=\mathrm{I} 25.00 \mathrm{~cm} & y=5.38 \mathrm{~cm} \\
I=20 \mathrm{cp} & x=\mathrm{I} 3 \mathrm{I} .97 \mathrm{~cm} & y=6.98 \mathrm{~cm}
\end{array}
$$

The complete curve from Io $\mathrm{cp}$ to $20 \mathrm{cp}$ may be seen in Fig. 2, which gives the form in which the blocks should be made. We used for the blocks an insulating material called "stabilit," made by the Allgemeine Electricitäts Gesellschaft of Berlin. This material 
is said to possess high insulation resistance combined with the ability to resist heat and moisture. It was used in preference to fiber because of the hygroscopic nature of the latter. Hard rubber would no doubt answer the purpose as well as stabilit.

Lack of uniformity in the wire or in winding may produce an accumulating error, so that it is desirable to test the resistance at a number of points as the wire is wound. In this connection it may be well to call attention to another way of considering the curve in Fig. 2, which is helpful in checking the resistance from point to point. Since the reading at II cp is to be $\frac{1}{1} \frac{0}{1}$ of the reading at IO $\mathrm{cp}$, the total resistance of the pressure circuit at this point should be $\frac{11}{10}$ of the resistance of the wattmeter, so that the added resistance should be $\frac{1}{10}$ the resistance of the wattmeter. Similarly the added resistance between II $\mathrm{cp}$ and $\mathrm{I} 2 \mathrm{cp}$, or between $\mathrm{I} 2 \mathrm{cp}$ and $\mathrm{I} 3 \mathrm{cp}$, should be $\frac{1}{10}$ the resistance of the wattmeter. Hence the resistance between points on each block corresponding to successive candlepowers should be equal to each other and equal to $\frac{1}{20}$ the resistance of the wattmeter. If greater accuracy is desired the resistance may be checked during the winding at every half candlepower point. Moreover, if slight errors are introduced in winding one coil, they may be partially neutralized in winding the other coil, so that the resultant error will be very small.

The wattmeter should be calibrated to read correctly as a wattmeter over the range for which it will be used, in order to avoid the necessity for applying a correction to the efficiency readings. It should have a compensating winding on the fixed coil, through which the potential current is carried in the opposite direction to the main current, so that the energy used in the potential circuit will not be measured along with that supplied to the lamp. This compensating winding is regularly supplied in low-range wattmeters by at least one well-known maker.

The reading of a wattmeter on direct current is affected by the earth's field. To eliminate the error from this source it is customary to take the mean of two readings, the direction of current flow through the circuits of the instrument being reversed for the second reading. Another way of accomplishing the same result is to balance the earth's field at the moving coil by the use of a small permanent magnet. This is the method we have used. A magnet 
Io $\mathrm{cm}$ long and I.3 cm diameter, when placed north and south at about five or six cm under the bottom of the wattmeter case balanced the earth's field almost exactly for all points of the scale that are used (see below), the wattmeter being placed with its longer dimension approximately north and south. Another way of eliminating the effect of the earth's field is to use an astatic wattmeter. Such instruments are not on the market in portable form, but they can doubtless be had from instrument makers if required. If the instrument is to be used in a location subject to strong and variable stray fields the use of an astatic wattmeter might be necessary, but in most cases the ordinary wattmeter with a compensating magnet will doubtless answer all requirements.

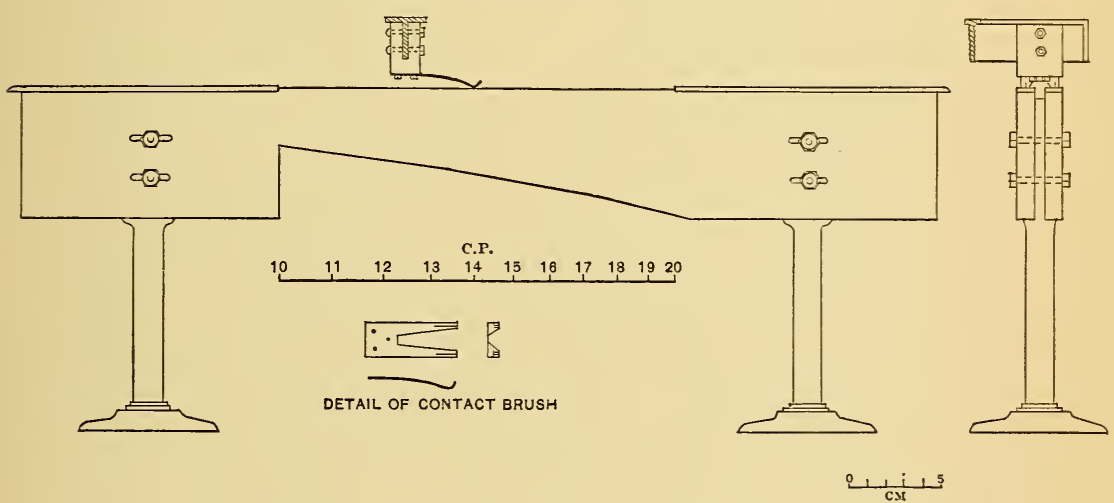

Fig. 2.-Blocks and Supports for Variable Rheostat.

Fig. 2 is the working drawing from which the blocks and supports were made. Although a range of from to to $20 \mathrm{cp}$ is sufficient, it is desirable to make the blocks longer, so that the wattmeter circuit will not be broken should the photometer move beyond these limits. On the top of each block, beyond the limits of the coil at each end, a copper strip was placed over which the contact brush moves when off the coil. The resistance wire at the end of the coil corresponding to Io $\mathrm{cp}$ was soldered directly into the copper strip at that end, but the other end of each coil was insulated from the metal strip beyond it. On each block the copper strip at one end was joined to the copper strip at the other end, so that the instrument reads watts per candle so long as the contact brush remains on the coil-that is, for all candlepower values between Io and 20, while 
beyond these limits the instrument reads watts. In order that for any position of the photometer the watts can be read directly, a switch is used by means of which the resistance external to the wattmeter can be short-circuited, so that either watts or efficiency can be read.

The blocks were mounted on stands with the tops of the coils at a convenient distance below the photometer carriage. On the latter was mounted a hard-rubber block carrying the phosphor bronze contact brush which completes the circuit from one coil to the other.

The blocks were slotted to receive bolts holding them in place on the stands. Thus a linear adjustment of each coil independently can be made, which is desirable. Fig. 3, which was made from a photograph, shows the blocks in position on the photometer, the wattmeter not being shown.

\section{EXPERIMENTAL RESULTS.}

The coils having been mounted and the wattmeter put in place, it was necessary to find the position of the small magnet in which it would neutralize the earth's field at the moving coil for the range used. By trial, using a I6-cp lamp, a position was found such that the wattmeter reading at one point was the mean of direct and reversed readings taken before the magnet was put in place. To test other points, the photometer was then set at 10,16 , and $20 \mathrm{cp}$, direct and reversed readings being made at each point. The agreement of the results is shown in Table I.

TABLE I.

\begin{tabular}{c|c|c}
\hline \multirow{2}{*}{ Photometer position } & \multicolumn{2}{|c}{ Wattmeter reading } \\
\cline { 2 - 3 } & Direct & Reversed \\
\hline $10 \mathrm{cp}$ & 49.3 & 49.4 \\
16 & 30.8 & 30.8 \\
20 & 24.6 & 24.6 \\
\hline
\end{tabular}

The next step consisted in placing the blocks in proper relation to the sliding contact to give a correct reading of watts per candle. To do this a I6-cp lamp was placed in the socket of the rotator, and 


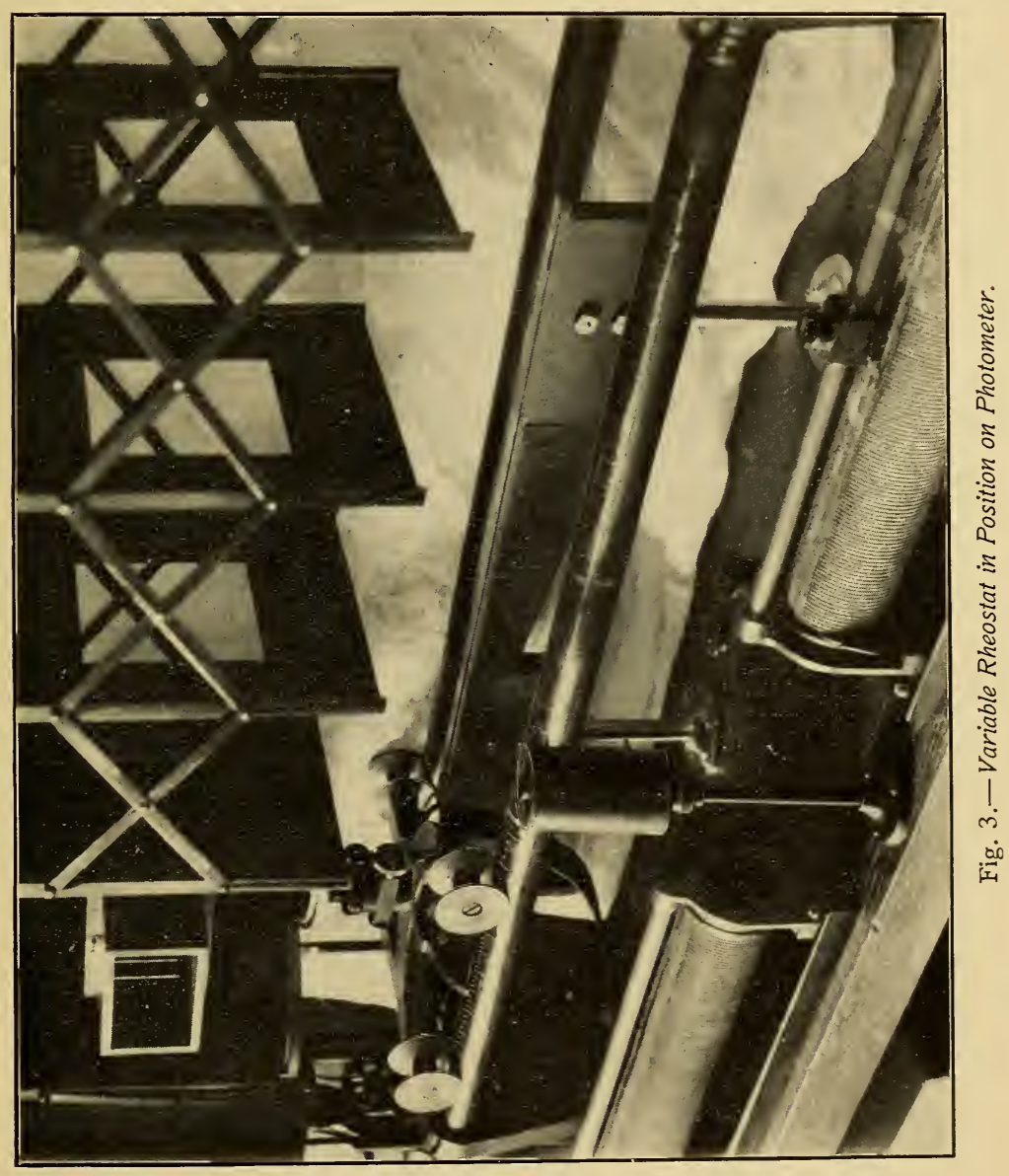


the screen was set at $\mathrm{r} 6 \mathrm{cp}$. The blocks were then adjusted until the wattmeter read the known efficiency of the lamp, and were then bolted to the supports. The efficiency meter was then in calibration for this one point. To check it at other points the lamp was kept at the original voltage, the photometer screen was placed successively at different positions on the bar, and readings were taken. The accuracy of the instrument at all points is shown in Table II, in which the first column contains the successive positions of the photometer on the bar. The second column gives the calculated efficiencies corresponding to these positions, as determined by dividing the watts supplied to the lamp by the values given in the first column. The third column contains the corresponding values observed on the efficiency meter.

TABLE II.

\begin{tabular}{c|c|c}
\hline Photometer position & Calculated efficiency & Observed efficiency \\
\cline { 2 - 3 } $10 \mathrm{cp}$ & 4.78 & 4.78 \\
11 & 4.34 & 4.34 \\
12 & 3.98 & 3.98 \\
13 & 3.68 & 3.67 \\
14 & 3.41 & 3.40 \\
15 & 3.18 & 3.18 \\
16 & 2.98 & 2.98 \\
17 & 2.81 & 2.80 \\
18 & 2.65 & 2.63 \\
19 & 2.51 & 2.51 \\
19.8 & 2.41 & 2.40 \\
\hline
\end{tabular}

It is thus seen that for I6-cp lamps ranging in efficiency from 2.4 to 4.8 watts per candle the apparatus gives true results to within less than I per cent. The instrument as adjusted for I6-cp lamps can be used without change for $32-\mathrm{cp}$ and 8-cp lamps. If we place a 32 -cp lamp in the comparison lamp socket, so that the photometer comes in the middle of the bar for 32 -cp test lamps, the efficiency of such lamps can be read by dividing the reading of the instrument by 20 instead of by ro. In our instrument the wattmeter has two scales, the upper with a range of O-I 50 watts, being used for I6-cp 
lamps. By using the lower scale of o-75 watts when measuring 32cp lamps the instrument is still direct reading. This refers only to the reading of the deflection; the electrical connections are the same for 16 and for 32 -cp lamps.

The accuracy of the instrument when used for 32-cp lamps depends on two things; first, whether the wattmeter is as accurate at the higher readings as at the ones used in measuring I6-cp lamps, and secondly, whether the earth's field is still balanced at the moving coil for the larger deflections. To test this point the observations given in Table III were made. A 32-cp lamp was placed in the test lamp socket and readings were made with the connections direct and reversed for different positions of the photometer, similar to the readings given in Table I for r6-cp lamps. The position of the small magnet was the same as before. The compensation for the earth's field is seen to be practically perfect.

TABLE III.

\begin{tabular}{c|r|c}
\hline \multirow{2}{*}{ Photometer position } & \multicolumn{2}{|c}{ Wattmeter reading } \\
\cline { 2 - 3 } & Direct & Reversed \\
\hline $20 \mathrm{cp}$ & 113.9 & 113.9 \\
32 & 70.8 & 70.8 \\
39.6 & 57.2 & 57.3 \\
\hline
\end{tabular}

In a similar way the same instrument can be used for 8-cp lamps by dividing the wattmeter readings by 5 . An objection to this is that the deflection being about one-half that for r6-cp lamps, the accuracy of reading is reduced. It is still, however, ample for commercial testing.

\section{APPLICATIONS.}

Besides the ordinary use of the instrument in reading watts per candle when lamps are tested for candlepower at some constant voltage, or for voltage at some fixed candlepower, it is very convenient for determining directly the voltage at which a lamp has a definite efficiency. Oftentimes, as in making life tests, it is desirable to know at what voltage the lamps have a given efficiency, say 3.I 
watts per candle. The only way to do this at present is to estimate the approximate voltage, and from the candlepower and wattage readings at that voltage to calculate the voltage corresponding to the required efficiency. This computation involves the use of the law of variation of efficiency with voltage, and is hence both tedious and unreliable. By the use of the efficiency meter, on the other hand, one operator gradually raises the voltage and observes the efficiency meter, while the other observer follows the change in candlepower with the photometer. When the instrument indicates the proper efficiency, the corresponding voltage is read. This method is not only quicker, but much more accurate and satisfactory.

If the utmost accuracy is desired, certain precautions must be taken. If the line voltage is considerably higher than the voltage at the lamp-that is, if there is a large drop through the rheostatsany change in the current will cause a relatively large change in the voltage on the lamp. Hence if the voltage at the lamp is adjusted when the photometer carriage is at Io $\mathrm{cp}$, the voltmeter will show a slight rise when the photometer is moved to $20 \mathrm{cp}$, due to the increase of resistance of the pressure circuit, and the consequent decrease in the pressure current. With the extreme travel of the carriage just given, this rise would be a few tenths of a volt, if the drop in the rheostats is ro volts and I6-cp lamps are being tested. In practice, however, there is almost never any occasion to move the photometer over more than three or four candlepower after the voltage has been adjusted, and moreover the effect of a motion of three or four candlepower at $16 \mathrm{cp}$ is not so great as at ro $\mathrm{cp}$, because of the higher resistance in the pressure circuit. A motion of the photometer from I4 to I $8 \mathrm{cp}$ would cause a rise in the voltage of about one-tenth of a volt, with Io volts drop through the rheostats. In the second place, the observer at the photometer would bring the screen to a position of approximate balance immediately and the second observer could correct for any small initial change, such initial change being rather common for other reasons. Finally, if the line voltage is kept but slightly above the lamp voltage, as may be done when a storage battery is used, the error becomes entirely negligible in ordinary cases. If the tests are made on a generator circuit where the voltage is unsteady it will usually be found desirable to use the "same circuit" method, in which case both lamps are changed about the same by 
the motion of the photometer, so that the error is negligible. This is the only source of error peculiar to the efficiency meter. The other sources of error are those which must be guarded against in any case. For example, whether we are measuring the current on an ammeter, the watts on a wattmeter, or the watts per candle on an efficiency meter, we must guard against errors due to the voltmeter current. If we read with the voltmeter in circuit we must correct the ammeter or wattmeter reading. In the case of the efficiency meter, either a correction can be applied, as for the other two instruments, or, if the meter is to be used for testing some one type of lamp, as I6-cp, IIo-volt, 3.5-watt-per-candle lamps, the coils can be moved slightly on the supports until the instrument reads correctly at $16 \mathrm{cp}$ with the voltmeter in circuit. The differential errors at other points on the scale will be negligibly small in commercial testing, and the efficiency of a lamp under test may be read at once, no corrections being necessary. This gives the efficiency meter an advantage over the ammeter or wattmeter as ordinarily used.

If we should set the voltage and then open the voltmeter switch, the voltage on the lamp would rise if there is any drop through the rheostats, so that the error in the efficiency, due to the change in current in the current circuit, would be much less important than the error in the intensity of the lamp, due to the rise in voltage. If the drop through the rheostats is small, so that the photometer error, due to opening the voltmeter switch, becomes negligible, the error in the efficiency can be eliminated by adjusting the coils with the voltmeter switch open, and then always reading the efficiency under the same conditions. It is necessary to make readings under the same conditions in any case, for even if the photometer error is eliminated by having the line voltage approximately equal to the lamp voltage, the reading of the ammeter or the wattmeter depends on whether the voltmeter is thrown on the test lamp or on the comparison lamp. Hence, to avoid errors, a routine must be followed, allowing proper corrections in case the voltmeter is left on the test lamp while the ammeter or the wattmeter is read.

We thus see that the efficiency meter, while subject to no greater errors than the ammeter or the wattmeter, is more flexible than either, and gives by a direct reading one of the most important constants of the incandescent lamp. 